\title{
A NEW TYPE OF CLUSTER SETS AND ITS APPLICATIONS
}

\author{
M. N. MUKHERJEE AND S. RAYCHAUDHURI
}

\begin{abstract}
In this paper we introduce the concept of a new type of cluster sets, termed $\delta$-cluster sets, of functions and multifunctions between topological spaces. Expressions of such sets are found and multifunctions with $\delta$-closed graphs are characterized. Also the behaviour of $\delta$-cluster sets toward $\delta$-continuity of a function is observed. Finally as applications, we find new characterizations of almost regularity, near compactness and near Lindelöfness of a topological space in terms of $\delta$-cluster sets of suitable multifunctions.
\end{abstract}

\section{Introduction}

The theory of cluster sets for arbitrary functions between topological spaces was initiated by Weston [15]. The theory has also been studied intensively by Joseph [7], Hamlett $[4,5]$ and many others. Joseph [7] has characterized compactness, Lindelöfness, $m$-compactness and $H$-closedness in terms of cluster sets and provided some consequences of these characterizations. There are some other well known spaces which have not yet been characterized in terms of cluster sets. This motivates us to introduce a new type of cluster sets called $\delta$-cluster sets in this paper. Our main objective of this paper is to find new characterizations of some other well known spaces in terms of $\delta$-cluster sets of suitable functions and multifunctions. We accomplish these for nearly compact, nearly Lindelöf and almost regular spaces with the help of $\delta$-cluster sets of certain types of multifunctions.

The next section is meant for quoting some definitions and results that are used in this paper. In Section 3 , we first find expressions for $\delta$-cluster sets of arbitrary functions between two toplogical spaces and then characterize multifunctions with $\delta$-closed graph in terms of $\delta$-cluster sets. Moreover, we investigate about degeneracy of a $\delta$-cluster set and use this as a tool to set new characterization of $\delta$-continuity of a function.

Received March 26, 1994.

1991 Mathematics Subject Classification. 54C60, 54C99, 54D10, 54D20.

Key words and phrases. $\delta$-cluster set, multifunction, nearly compact space, nearly Lindelöf space, almost regularity. 
In the last section we bring out some applications of the concepts developed in the earlier section by achieving new characterizations of nearly compact, nearly Lindelöf and almost regular spaces.

\section{Preliminaries}

Throughout the present paper $X$ and $Y$ always denote topological spaces. We denote the closure and interior of a subset $A$ of a space $X$ by $\operatorname{cl} A$ and int $A$ respectively. A point $x \in X$ is said to be a $\delta$-adherent point of a set $A$ in $X$ [14] if $A \cap U \neq \Phi$ for every regular open set $U$ containing $x$, or equivalently, $A \cap$ intcl $V \neq \Phi$ for every open neighbourhood (henceforth nbd, for short) of $x$. The set of all $\delta$-adherent points of $A$ is called the $\delta$-closure of $A$ [14], to be denoted by $\delta \operatorname{cl} A$. $A$ is called $\delta$-closed if $A=\delta \operatorname{cl} A$. The complement of a $\delta$-closed set is called $\delta$-open. The $\delta$-open sets in a space $X$ form a topology on $X$, called $\delta$-topology on $X$, for which the regular open sets form a base [2]. The collection of all $\delta$-open sets of $X$ (resp. $Y$ ) is denoted by $\delta O(X)$ (resp. $\delta O(Y)$ ). For a set $A$ in a space $X$, the collection of all $\delta$-open sets containing $A$ will be denoted as $\Delta(A)$. In particular, when $A=\{x\}(x \in X)$, we shall write $\Delta(x)$ for $\Delta(\{x\})$. When $A \subset Y$, we continue to write $\Delta(A)$ to mean the collection of all $\delta$-open sets of $Y$, each containing $A$, and hope that the context will leave no scope for confusion. We say that a point $x \in X$ is in the $\theta$-closure [14] of a set $A(\subset X)$, written $x \in \theta \operatorname{cl} A$, if each open $\operatorname{nbd} U$ of $x$ satisfies $A \cap \operatorname{cl} U \neq \Phi$. $A$ is called $\theta$-closed [14] if $\theta \operatorname{cl} A=A$. It is known [14] that for an open set $A$ in a space $X, \theta \operatorname{cl} A=\delta \operatorname{cl} A=\operatorname{cl} A$. $A$ space $X$ is said to be almost regular [12] if for each regular closed set $F$ in $X$ and each point $x$, not contained in $F$, there exist disjoint open sets $U$ and $V$ such that $x \in U$ and $F \subset V$, equivalently, if every regular-closed set $F$ in $X$ is expressible as an intersection of some regular-closed nbds of $F$.

Let $\mathcal{F}$ be a filter (or a filterbase) on a space $X$. A point $x \in X$ is called a $\delta$-adherent point of $\mathcal{F}$, written as $x \in \delta-a d \mathcal{F}$, if $x \in \cap\{\delta \mathrm{cl} F: F \in \mathcal{F}\} ; \mathcal{F}$ is said to $\delta$-converge to $x$, denoted by $\mathcal{F} \stackrel{\delta}{\longrightarrow} x$, if for each open nbd $U$ of $x$ there is an $F \in \mathcal{F}$ such that $F \subset$ intcl $U$ [10]. A subset $A$ of $X$ is called $N$-closed relative to $X$ [3] if every cover of $A$ by regular open (or $\delta$-open) sets of $X$ has a finite subcover for $A$. If, in addition, $A=X$ then $X$ is called a nearly compact spaces [13]. The following results will be used in the sequel.

Lemma 2.1 (a) [13] Every regular closed subset of a nearly compact space is $N$-closed relative to $X$.

(b) [8] A space $X$ is nearly compact iff $\delta-a d \mathcal{F} \neq \Phi$, for every filterbase $\mathcal{F}$ on $X$.

We shall represent the class of multifunctions from a space $X$ to a space $Y$ by $M(X, Y)$, where by a multifunction $F: X \rightarrow Y$ we mean a function from $X$ to $\mathcal{P}(Y)-$ $\{\Phi\}, \mathcal{P}(Y)$ being the power set of $Y$.

\section{3. $\delta$-cluster Sets of Functions and Multifunctions}


Definition 3.1. Let $F: X \rightarrow Y$ be a multifunction, and $x \in X$. Then the $\delta$-cluster set, denoted by $\mathcal{D}(F, x)$, of $F$ at the point $x$ is given by the set $\cap\{\delta \mathrm{cl} F(U)$ : $U \in \Delta(x)\}$, the $\delta$-cluster set of a function (i.e. single valued function) $f: X \rightarrow Y$ at a point $x \in X$ is defined in the same way viz. $\mathcal{D}(f, x)=\cap\{\delta \mathrm{cl} f(U): U \in \Delta(x)\}$.

In order to characterize $\delta$-cluster sets we need the following lemma, the proof of which is quite straightforward.

Lemma 3.2. A filter (or fiiterbase) on a space $X \delta$-adheres at a point $x \in X$ iff there is a filter $\mathcal{Y}$ on $X$, finer than $\mathcal{F}, \delta$-converging to $x$.

Theorem 3.3. For a function $f: X \rightarrow Y$ the following statements are equivalent:

(a) $y \in \mathcal{D}(f, x)$

(b) There is a filter $\mathcal{F}$ on $X$ such that $\mathcal{F} \stackrel{\delta}{\longrightarrow} x$ and $f(\mathcal{F}) \stackrel{\delta}{\longrightarrow} y$.

(c) $f^{-1}(\Delta(y)) \delta$-adheres at $x$.

Proof. (a) $\Rightarrow(\mathrm{b})$ : Since $y \in \mathcal{D}(f, x)$, we have $V \cap f(U) \neq \Phi$ for every $V \in \Delta(y)$ and each $U \in \Delta(x)$. Obviously, $f^{-1}(V) \cap U \neq \Phi$ and $f^{-1}(\Delta(y)) \delta$-adheres at $x$. In view of the above lemma, there is a filter $\mathcal{Y}$ on $X$ finer than $f^{-1}(\Delta(y))$ such that $\mathcal{Y} \stackrel{\delta}{\longrightarrow} x$. If possible, let there exist $V \in \Delta(y)$ such that $f(G) \not \subset V$, for every $G \in \mathcal{Y}$. Then $f(G) \cap(Y-V) \neq \Phi$ for each $G \in \mathcal{Y}$. In particular, $f^{-1}(V) \in \mathcal{Y}$ so that $f f^{-1}(V) \cap(Y-V) \neq \Phi$ which is absurd. Hence $f \mathcal{Y} \stackrel{\delta}{\longrightarrow} y$.

$(\mathrm{b}) \Rightarrow(\mathrm{c})$ : Let $\mathcal{F}$ be a filter on $X \delta$-converging to $x$ and $f(\mathcal{F}) \delta$-converge to some point $y$ in $Y$. Let $V$ be any $\delta$-open nbd of $y$ in $Y$. Then there is $F \in \mathcal{F}$ such that $f(F) \subset V$. We then have $F \subset f^{-1}(V)$ and hence $f^{-1} V \in \mathcal{F}$. Thus $\mathcal{F}$ is finer than $f^{-1}(\Delta(y))$. Since $\mathcal{F} \stackrel{\delta}{\longrightarrow} x$, it follows by Lemma 3.2 that $f^{-1}(\Delta(y))$ must $\delta$-adhere at $x$.

(c) $\Rightarrow\left(\right.$ a): Let $f^{-1}(\Delta(y)) \stackrel{\delta}{\longrightarrow} x$. Then for every $V \in \Delta(y)$ and $U \in \Delta(x)$, we have $f^{-1}(V) \cap U \neq \Phi$ and hence $V \cap f(U) \neq \Phi$. Thus $y \in \mathcal{D}(f, x)$.

Next, we want to use cluster set as a tool to characterize multifunctions with $\delta$ closed graph and $\delta$-continuity of a function. To that end, we need the following lemma which incidentally gives yet another characterization of the $\delta$-cluster set of a function at a point.

Lemma 3.4. Let $F: X \rightarrow Y$ be a multifunction and $x \in X$. Then $p_{2}[(\{x\} \times$ $Y) \cap \delta c l G(F)]=\mathcal{D}(F, x)$, where $p_{2}: X \times Y \rightarrow Y$ denotes the projection map, and $G(F)$ is the graph of $F$.

Proof. Let $y \in \mathcal{D}(F, x)$ and $G$ be any $\delta$-open set in $X \times Y$ containing $(x, y)$. Then by Proposition 4 of [6], $G=U \times W$ where $x \in U \in \Delta(x), y \in W \in \Delta(y)$. Since $y \in \delta \mathrm{cl} F(U)$, we have $W \cap F(U) \neq \Phi$. Hence $(U \times W) \cap G(F) \neq \Phi$, i.e., $(x, y) \in \delta \mathrm{cl} G(F)$ and $y \in p_{2}[(\{x\} \times Y \cap \delta \mathrm{cl} G(F)]$. Reversing the above arguments we get the reverse inclusion. 
Theorem 3.5. Let $F: X \rightarrow Y$ be a multifunction. Then the following are equivalent:

(a) $F$ has a $\delta$-closed graph.

(b) $F(x)=p_{2}[(\{x\} \times Y) \cap \delta c l G(F)]$, for each $x \in X$.

(c) For each $x \in X, \mathcal{D}(F, x)=F(x)$.

Proof. '(a) $\Rightarrow(\mathrm{b})$ ' is obvious, and '(b) $\Rightarrow$ (c)' follows immediately from the above lemma. We thus only prove '(c) $\Rightarrow$ (a)' as follows. Let $(x, y) \in X \times Y-G(F)$. Then $y \notin F(x)$ so that $y \notin \mathcal{D}(F, x)$. Then there are a $\delta$-open set $V$ containing $y$ and a $U \in \Delta(x)$ such that $V \cap F(U)=\Phi$. Hence $(U \times V) \cap G(F)=\Phi$. Now, $U \times V \in \Delta(x, y)$ and we can conclude that $G(F)$ is $\delta$-closed.

The above theorem shows that multifunctions with $\delta$-closed graphs can be characterised in terms of $\delta$-cluster sets. In particular, when $f$ is a single-valued function, then the theorem asserts that $\delta$-closedness of the graph of $f$ can be characterized by the fact of its $\delta$-cluster set at each point being degenerate. The next two theorems give yet other two situations when the $\delta$-cluster sets of certain classes of functions become degenerate. We recall in this connection that a function $f: X \rightarrow Y$ is $\delta$-closed if $F(A)$ is $\delta$-closed in $Y$ for each $\delta$-closed set $A$ in $X$.

Theorem 3.6. Let $f$ be a $\delta$-closed map from an almost regular space $X$ into a space $Y$. If $f^{-1}(y)$ is $\delta$-closed in $X$ for every $y \in Y$, then $\mathcal{D}(f, x)$ is degenerate for every $x \in X$.

Proof. It is class that $\mathcal{D}(f, x)=\cap\{\delta \mathrm{cl} f(U): U \in \Delta(x)\} \subset \cap\{\delta \mathrm{cl} f(\delta \mathrm{cl} U): U \in$ $\Delta(x)\}=\cap\{f(\delta \mathrm{cl} U): U \in \Delta(x)\}$ (as $f$ is $\delta$-closed). Let $y \in Y$ with $y \neq f(x)$. Since $X$ is almost regular and $f^{-1}(y)$ is $\delta$-closed with $x \notin f^{-1}(y)$, there is a $\delta$-open set $U$ such that $x \in U$ and $f^{-1}(y) \cap \delta \operatorname{cl} U=\Phi$, which shows that $y \notin f(\delta \mathrm{cl} U)$ and consequently, $y \notin \mathcal{D}(f, x)$. Hence $\mathcal{D}(f, x)$ is degenerate.

Theorem 3.7. Let $f$ be a $\delta$-closed injection from a Hausdorff space $X$ into a space $Y$, then $\mathcal{D}(f, x)$ is degenerate for every $x \in X$.

Proof. For any $x \in X$, we have as in the above proof, $\mathcal{D}(f, x) \subset \cap\{f(\delta \mathrm{cl} U)$ : $U \in \Delta(x)\}$. Suppose $x, x_{1} \in X$ with $x \neq x_{1}$, then $f(x) \neq f\left(x_{1}\right)$. Since $X$ is Hausdorff, there exist open nbds $U$ and $V$ of $x$ and $x_{1}$ respectively such that $U \cap V=\Phi$ and hence intcl $U \cap \operatorname{intcl} V=\Phi$. Then $\delta \operatorname{cl} W \cap V=\Phi$, where $W=\operatorname{intcl} U \in \Delta(x)$. Hence $x_{1} \notin \delta \mathrm{cl} W$ so that $f\left(x_{1}\right) \notin f(\delta \mathrm{cl} W)$. Thus $f\left(x_{1}\right) \notin \mathcal{D}(f, x)$. Consequently $\mathcal{D}(f, x)=\{f(x)\}$.

Definition 3.8. [11] A function $f: X \rightarrow Y$ is $\delta$-continuous if for each $x \in X$ and each regular open (or $\delta$-open) set $V$ containing $f(x)$, there exists a regular open (or $\delta$-open) set $U$ containing $x$ such that $f(U) \subset V$, or equivalently, for every regularly open set $V$ in $Y, f^{-1}(V)$ is $\delta$-open in $X$.

We show in the following theorem that the condition of degeneracy of the $\delta$-cluster 
set of a function at a point $x$ characterizes the $\delta$-continuity of the function at $x$, under certain restriction on the codomain space of the function.

Theorem 3.9. Let $f: X \rightarrow Y$ be a mapping from a space $X$ into a nearly compact Hausdorff space $Y$ and $x \in X$. Then $f$ is $\delta$-continuous at $x$ iff $\mathcal{D}(f, x)$ is degenerate.

Proof. Let $f: X \rightarrow Y$ be not $\delta$-continuous at a point $x \in X$, where $Y$ is a nearly compact space. Then there is a regularly open set $V$ containing $f(x)$ such that $f(U) \cap(Y-V) \neq \neq \Phi$, for every $\delta$-open set $U$ containing $x$. Now, $\{\delta$ cl $f(U) \cap(Y-V)$ : $U \in \Delta(x)\}$ is a family of $\delta$-closed sets and has the finite intersection property. $X$ being nearly compact, we have by Lemma 3.2 that $\cap\{\delta$ cl $f(U) \cap(Y-V): U \in \Delta(x)\} \neq \Phi$, i.e. $\mathcal{D}(f, x) \cap(Y-V) \neq \Phi$. Hence $\mathcal{D}(f, x)$ is not degenerage.

Conversely, assume that $f$ is $\delta$-continuous at a point $x \in X$. Let $y \in Y$ with $f(x) \neq y$. Since $Y$ is Hausdorff, there are regular open sets $U_{f(x)}$ and $U_{y}$ containing $f(x)$ and $y$ respectively such that $U_{f(x)} \cap U_{y}=\Phi$. Since $f$ is $\delta$-continuous at $x$, there is a $\delta$-open set $V_{x}$ in $X$ containing $x$ such that $f\left(V_{x}\right) \subset U_{f(x)}$. So, $f\left(V_{x}\right) \cap U_{y}=\Phi$. Consequently, $y \notin \delta \mathrm{cl} f\left(V_{x}\right)$ and $\{f(x)\}=\cap\{\delta \operatorname{cl} f(U): U \in \Delta(x)\}=\mathcal{D}(f, x)$. Hence $\mathcal{D}(f, x)$ is degenerate.

Definition 3.10. [1] A function $f: X \rightarrow Y$ is said to be $\delta^{*}$-continuous if for every open set $V$ containing $f(x)$, there is a regular open set $U$ containing $x$ such that $f(U) \subset V$.

Theorem 3.11. If $f: X \rightarrow Y$ is a $\delta^{*}$-continuous function then $\delta c l\{f(x)\} \subset$ $\mathcal{D}(f, x) \subset \theta \operatorname{cl}\{f(x)\}$.

Proof. $\delta \mathrm{cl}\{f(x)\} \subset \mathcal{D}(f, x)$ is obvious:

Suppose $y \in \mathcal{D}(f, x)$ but $y \notin \theta \operatorname{cl}\{f(x)\}$. Then there is an open nbd $V$ of $y$ such that $f(x) \notin \mathrm{cl} U$. Hence there is an open set $V$ such that $f(x) \in V$ and $U \cap V=\Phi$. Since $f$ is $\delta^{*}$-continuous, there exists a regular open set $W$ such that $x \in W$ and $f(W) \subset V$. Since $y \in \mathcal{D}(f, x), y \in \delta \mathrm{cl} f(W)$ (since $W$ is also $\delta$-open) $\subset \delta \operatorname{cl} V=\operatorname{cl} V$. Thus $U \cap V \neq \Phi$, a contradiction. Therefore, $\mathcal{D}(f, x) \subset \theta \mathrm{cl}\{f(x)\}$.

\section{Applications}

As proposed earlier, the purpose of this section is to highlight some applications of the concept of $\delta$-cluster sets by presenting certain new characterizations of some well known classes of spaces via the notion of $\delta$-cluster sets. We start with the case of almost regularity, and introduce the following definition as a prerequisite.

Definition 4.1. A multifunction $F: X \rightarrow Y$ is called upper $\delta$-continuous if for each $\delta$-open set $V$ containing $F(x)$, there is a $\delta$-open set $U$ containing $x$ such that $F(U) \subset V$. 
Theorem 4.2. A space $Y$ is almost regular iff $\mathcal{D}(F, x)=\delta c l F(x)$ for each $x \in X$ and each upper $\delta$-continuous multifunction $F$ from any space $X$ to $Y$.

Proof. Let $Y$ be an almost regular space and $F$ an upper $\delta$-continuous function from a space $X$ to $Y$. It is obviously true that $\delta$ cl $F(x) \subset \mathcal{D}(F, x)$. Now, if $x \in X$ and $W$ is a $\delta$-open set containing $F(x)$ in $Y$, then by upper $\delta$-continuity of $F$ there exists a $\delta$-open set $V$ containing $x$ in $X$ such that $F(V) \subset W$. Hence

$$
\mathcal{D}(F, x)=\cap\{\delta \mathrm{cl} F(V): V \in \Delta(x)\} \subset \cap\{\delta \mathrm{cl} W: W \in \Delta(F(x))\}=\cap\{\mathrm{cl} W: W \in \Delta(F(x))\} .
$$

It now suffices to show that $\cap\{\operatorname{cl} W: W \in \Delta(F(x))\} \subset \delta \mathrm{cl} F(x)$ as $\delta \mathrm{cl} F(x) \subset \cap\{\mathrm{cl} W$ : $W \in \Delta(F(x))\}$ is obvious.

Let $y \in \cap\{\mathrm{cl} W: W \in \Delta(F(x))\}$

If $y \notin \delta \mathrm{cl} F(x)$, then since $Y$ is almost regular, there are disjoint open sets $U, V$ such that $y \in U$ and $\delta \mathrm{cl} F(x) \subset V$. Put $V_{1}=\operatorname{intcl} V$, then $V_{1}$ is a $\delta$-open set containing $\delta$ cl $F(x)$ such that $\operatorname{cl} V_{1} \cap U=\operatorname{cl} V \cap U=\Phi$. Since $F(x) \subset V_{1}$ we have by (1), $y \in \operatorname{cl} V_{1}$. Also $y \in U$ so that we get $U \cap V_{1} \neq \Phi$, a contradiction. Hence $\mathcal{D}(F, x)=\delta$ cl $F(x)$.

Conversely, suppose $\left(Y, \tau_{Y}\right)$ is not almost regular. Then there is a nonempty regular closed set $M \subset Y$ such that $\cap\{\delta c l W: M \subset W \in \delta O(Y)\} \supsetneqq M$. Choose $x_{0} \in M$ and let $\mathcal{F}=\{F \in \delta O(Y): M \subset F\}$. Now, $\mathcal{F}\left(x_{0}\right)=\left\{V \subset Y: x_{0} \notin V\right\} \cup\left\{V \in \delta O(Y): x_{0} \in V\right.$ and $F \subset V$ for some $F \in \mathcal{F}\}$ forms a base for some topology $\tau$ on $Y$. Set $X=Y$ and define a multifunction $\psi:(X, \tau) \rightarrow\left(Y, \tau_{Y}\right)$ by

$$
\psi(x)=M \cup\{x\}, \quad \text { for } \quad x \neq x_{0} .
$$

and

$$
\psi\left(x_{0}\right)=M
$$

We show that $\psi$ is upper $\delta$-continuous on $X$. Let $V$ be a $\delta$-open set in $\left(Y, \tau_{Y}\right)$ such that $\psi\left(x_{0}\right) \subset V$. Since $M \subset V \in \delta O(Y), V \in \tau$ and also $V$ is $\tau$-closed. Thus $V$ is a regular open and hence a $\delta$-open set in $(X, \tau)$ containing $x_{0}$ such that $\psi(V)=M \cup \psi\left(V-\left\{x_{0}\right\}\right)=$ $M \cup\left(V-\left\{x_{0}\right\}\right) \subset V$. Next, let $x \in X-\left\{x_{0}\right\}$ and $V$ a $\delta$-open nbd of $\psi(x)$ in $\left(Y, \tau_{Y}\right)$. Consider $U=\tau-\operatorname{int}(\tau-\operatorname{cl}\{x\})$. Then $U$ is a regular open and hence a $\delta$-open set in $(X, \tau)$ containing $x$ such that $\psi(U) \subset \psi\left(\left\{x_{0}, x\right\}\right)=\{x\} \cup M \subset V$. Thus $\psi$ is upper $\delta$-continuous on $X$.

Next we show that $\cap\left\{\delta \mathrm{cl} \psi(V): x_{0} \in V \in \delta O(X)\right\}=\cap\{\delta \mathrm{cl} W: M \subset W \in \delta O(Y)\}$. In fact, let $t \in \cap\{\delta \mathrm{cl} W: M \subset W \in \delta O(Y)\}$, and let $V \in \delta O(X)$ such that $x_{0} \in V$. Then there is $V_{1} \in \mathcal{F}\left(x_{0}\right)$ with $x_{0} \in V_{1} \subset V$. Thus $M \subset V_{1} \in \delta O(Y)$ so that $t \in \delta$ cl $V_{1}$ in $Y$. Hence $t \in \delta \mathrm{cl} V=\delta \mathrm{cl} \psi(V)$ in $Y$, proving that $t \in \cap\left\{\delta \mathrm{cl} \psi(V): x_{0} \in V \in \delta O(X)\right\}$. Again, for any $W \in \delta O(Y)$ with $M \subset W$, since $\psi\left(x_{0}\right)=M \subset W$ there exists, by upper $\delta$-continuity of $\psi$, a $V \in \delta O(X)$ with $x_{0} \in V$ such that $\psi(V) \subset W$. Hence the proposed equality is established. It then follows that $\mathcal{D}\left(\psi, x_{0}\right) \neq M=\delta$ cl $\psi\left(x_{0}\right)$. This completes the proof. 
Definition 4.3. If $X$ and $Y$ are spaces, $A \subset X$ and $F \in M(X, Y)$, we define $\mathcal{D}(F, A)=\bigcup_{x \in A} \mathcal{D}(F, x)$.

Lemma 4.4. A set $A$ in a space $X$ is $N$-closed relative to $X$ iff whenever for each filterbase $\mathcal{F}$ on $X$ such that $F \cap c \neq \Phi$ is satisfied for each $F \in \mathcal{F}$ and each $c \in \Delta(A)$, we have $A \cap \delta-a d \mathcal{F} \neq \Phi$.

Proof. Let $A(\subset X)$ be $N$-closed relative to $X$ and $\mathcal{F}$ be a filterbase on $X$ with the stated property. If possible, suppose that $A \cap \delta-a d \mathcal{F}=\Phi$. Then for each $x \in A$ there are a regular open set $V(x)$ containing $x$ and an $F(x) \in \mathcal{F}$ such that $V(x) \cap F(x)=\Phi$. By $N$-closedness of $A$ relative to $X, A \subset \cup\left\{V(x): x \in A^{*}\right\}$, for some finite subset $A^{*}$ of $A$. Choose $F^{*} \in \mathcal{F}$ with $F^{*} \subset \bigcap_{x \in A^{*}} F(x)$. Then $F^{*} \cap\left[\bigcup_{x \in A^{*}} V(x)\right]=\Phi$ and $\bigcup_{x \in A^{*}} V(x) \in \Delta(A)$. Hence $\mathcal{F}$ does not satisfy the condition of the lemma.

Conversely, assume that $A$ is not $N$-closed relative to $X$. Then there is an open cover $\left\{U_{\alpha}: \alpha \in \Lambda\right\}$ of $A$ such that $A \not \subset \cup\left\{\operatorname{intcl} U_{\alpha}: \alpha \in \Lambda_{0}\right\}$, for every finite subset $\Lambda_{0}$ of $\Lambda$. Thus $\mathcal{F}=\left\{A-\bigcup_{\alpha \in \Lambda_{0}} \operatorname{intcl} U_{\alpha}: \Lambda_{0}\right.$ is a finite subset of $\left.\Lambda\right\}$ is a filterbase on $X$ such that $F \cap C \neq \Phi$, for each $F \in \mathcal{F}$ and each $C \in \Delta(A)$, but $A \cap \delta-\operatorname{ad\mathcal {F}}=\Phi$.

Theorem 4.5. For a space $X$, the following are equivalent:

(a) $X$ is nearly compact.

(b) $\mathcal{D}(F, A)=\delta-a d F(\Delta(A))$, for each space $Y$, each multifunction $F$ from $X$ to $Y$ and each regular closed set $A$ in $X$.

(c) $\mathcal{D}(F, A)$ is $\delta$-closed in $Y$, for each space $Y$, each multifunction $F$ from $X$ to $Y$ and each regular closed set $A$ in $X$.

Proof. $(\mathrm{a}) \Rightarrow(\mathrm{b})$ : Let $Y$ be any given space and $A$ be a regular closed set in $X$, where $X$ is nearly compact. For each $x \in A, \Delta(A) \subset \Delta(x)$. If $F$ is any multifunction from $X$ to $Y$, we have $F(\Delta(A)) \subset F(\Delta(x))$. Then $\mathcal{D}(F, x)=\cap\{\delta$ cl $F(U): U \in \Delta(x)\} \subset$ $\cap\{\delta \mathrm{cl} F(W): W \in \Delta(A)\}$. Hence $\mathcal{D}(F, A) \subset \delta-\operatorname{ad} F(\Delta(A))$.

Now, by Lemma 2.1 (a), $A$ is $N$-closed relative to $X$. Let $z \in \delta-a d F(\Delta(A))$. Thus for each $V \in \Delta(z)$ and $W \in \Delta(A)$ in $X$, we have $V \cap F(W) \neq \Phi$ so that $F^{-}(V) \cap W \neq \Phi$, where, as usual, $F^{-}(B)=\{x \in X: F(x) \cap B \neq \Phi\}$ for any $B \subset Y$. Since $F^{-}(\Delta(z))$ is a filterbase on $X$, we have by Lemma 4.4, $A \cap \delta-a d F^{-}(\Delta(z)) \neq t \Phi$. Thus for each $x \in A \cap \delta-a d F^{-}(\Delta(z))$, we have $W \cap F^{-}(V) \neq \Phi$, for each $W \in \Delta(x)$ and $V \in \Delta(z)$. Hence $F(W) \cap V \neq \Phi$, for all such $W$ and $V$. Therefore, $z \in \delta \operatorname{cl} F(W)$ for each $W \in \Delta(x)$ and hence $z \in \mathcal{D}(F, x) \subset \mathcal{D}(F, A)$.

(b) $\Rightarrow(\mathrm{c})$ : Obvious.

(c) $\Rightarrow($ a $):$ Let $\mathcal{F}$ be a filterbase on $X$. We only show that $\delta$-ad $\mathcal{F} \neq \Phi$ (see Lemma 2.1 (b)). Suppose $y_{0} \notin X$ and let $Y=X \cup\left\{y_{0}\right\}$, and $\tau_{y}=\left\{U \subset Y: y_{0} \notin U\right\} \cup\left\{U \subset Y: y_{0} \in\right.$ $U$ and $F \subset U$ for some $F \in \mathcal{F}\}$. Then $\tau_{Y}$ is a topology on $Y$. Consider the identity map $f: X \rightarrow\left(Y, \tau_{Y}\right)$. Now, $X$ is $\delta$-closed in $X$ and hence by hypothesis, $\mathcal{D}(f, X)$ is $\delta$-closed in $\left(Y, \tau_{Y}\right)$. We find that $y_{0} \in \delta \operatorname{cl}(\mathcal{D}(f, X))=\mathcal{D}(f, X)$. Hence there is $x_{0} \in X$ such that 
$y_{0} \in \mathcal{D}\left(f, x_{0}\right)$. Then for every $\delta$-open set $V$ in $X$ containing $x_{0}$ and each $\delta$-open set $W$ containing $y_{0}, f(V) \cap W \neq \Phi$, i.e., for each $F \in \mathcal{F}$ and each $\delta$-open set $V$ in $X$ containing $x_{0}$, we have $F \cap f(V) \neq \Phi$ (we notice that for each $F \in \mathcal{F}, F \cup\left\{y_{0}\right\} \in \Delta\left(y_{0}\right)$ ). Thus $F \cap V \neq \Phi$. Hence $x_{0} \in \delta-a d \mathcal{F}$ in $X$ so that $\delta-a d \mathcal{F} \neq \Phi$.

From the above proof it follows that

Corollary 4.6. Let $F \in M(X, Y)$. If $A \subset X$ is $N$-closed relative to $X$, then $\mathcal{D}(F, A)=\delta \operatorname{cl} \mathcal{D}(F, A)=\delta-\operatorname{ad} F(\Delta(A))$.

Corollary 4.7. Let $F \in M(X, Y)$ and $F$ have a $\delta$-closed graph. Then $F(A)$ is $\delta$-closed in $Y$ for each $N$-closed set $A$ relative to $X$.

Proof. We have $\mathcal{D}(F, A)=\bigcup_{x \in A} \mathcal{D}(F, x)=\bigcup_{x \in A} F(x)$ (by Theorem 3.5) $=F(A)$. Then in view of Corollary 4.6, $F(A)$ becomes $\delta$-closed.

Definition 4.8. [9] A space $X$ is called nearly Lindelöf if every regular open cover of $X$ has a countable subcover, a subset $A$ of $X$ is called nearly Lindelöf relative to $X$ if every cover of $A$ by regular open sets of $X$ has a countable subcover for $A$.

Theorem 4.9. A space $X$ is nearly Lindelöf iff every cover of $X$ by $\delta$-open sets of $X$ has a countable subcover.

Proof. Follows easily from the facts that every regular open set is a $\delta$-open set, and the regular open sets form a base for the $\delta$-topology on $X$.

Theorem 4.10. A $\delta$-closed subset of a nearly Lindelöf space $X$ is nearly Lindelöf relative to $X$.

Proof. Straightforward.

Theorem 4.11. A space $X$ is nearly Lindelöf iff every filterbase on $X$ with countable intersection property has a $\delta$-adherent point.

Proof. Let $\mathcal{F}$ be a filterbase on a nearly Lindelöf space $X$ with the countable intersection property. If possible, let $\delta-a d \mathcal{F}=\Phi$. Then for each $x \in X$, there are a regular open set $U_{x}$ containing $x$ and an $F_{x} \in \mathcal{F}$ such that $U_{x} \cap F_{x}=\Phi$. Now, $\left\{U_{x}: x \in X\right\}$ is a regular open cover of $X$, so that $X=\bigcup_{x \in X_{0}} U_{x}$ for a countable subset $x_{o}$ of $x$. It is clear that

$$
\left(\bigcup_{x \in X_{0}} U_{x}\right) \cap\left(\bigcap_{x \in X_{0}} F_{x}\right)=\Phi \text {, i.e., } \bigcap_{x \in X_{0}} F_{x}=\Phi \text {, a contradiction to the countable }
$$
intersection property of $\mathcal{F}$.

Conversely, let $\mathcal{U}=\left\{U_{\alpha}: \alpha \in \Lambda\right\}$ be a regular open cover of $X$ having no countable subcover. Then $\mathcal{F}=\left\{X-\bigcup_{\alpha \in \Lambda_{0}} U_{\alpha}: \Lambda_{0}\right.$ is a countable subset of $\left.\Lambda\right\}$ is a filterbase on $X$ with countable intersection property. Hence there exists $x_{0} \in \delta-a d \mathcal{F}$. Now, $x_{0} \in U_{\beta} \in \mathcal{U}$ for some $\beta \in \Lambda$. Since $X-U_{\beta} \in \mathcal{F}$, it follows that $x_{0} \notin \delta$ cl $\left(X-U_{\beta}\right) \supset \delta-a d \mathcal{F}$.

Lemma 4.12. A subset $A$ of a space $X$ is nearly Lindelöf relative to $X$ iff 
for each filterbase $\mathcal{F}$ on $X$ such that $I \cap V \neq \Phi$ is satisfied by each $V \in \Delta(A)$ and every countable intersection $I$ of elements of $\mathcal{F}$, one has $A \cap \delta-a d \mathcal{F} \neq \Phi$.

Proof. The proof, being similar to that of Lemma 4.4, is omitted.

Definition 4.13. A space $X$ is called a $D$-space if each countable intersection of $\delta$-open sets in $X$ is $\delta$-open in $X$.

Theorem 4.14. For a space $X$, the following are equivalent:

(a) $X$ is nearly Lindelöf.

(b) $\mathcal{D}(F, A)=\delta-\operatorname{ad}(F(\Delta(A)))$, for any multifunction $F$ from $X$ to any $D$-space $Y$ and any regular closed (or $\delta$-closed) subset $A$ of $X$.

(c) $\mathcal{D}(F, A)$ is $\delta$-closed in $Y$, for any multifunction $F$ from $X$ to any $D$-space $Y$ and any regular closed (or $\delta$-closed) subset $A$ of $X$.

Proof. $(\mathrm{a}) \Rightarrow(\mathrm{b})$ : It is clear that $\mathcal{D}(F, A) \subset \delta-a d F(\Delta(A))$

Next, let $z \in \delta-a d F(\Delta(A))$. Clearly $\Delta(z)$ is a filter on $Y$, so that $F^{-}(\Delta(z))(=\mathcal{F}$, say) is a filterbase on $X$. Let $\left\{F^{-}\left(W_{i}\right): i=1,2, \ldots\right\}$ be a countable collection of members of $\mathcal{F}$, where each $W_{i} \in \Delta(z)$. Since $Y$ is a $D$-space, $\bigcap_{i=1}^{\infty} W_{i}=W$ (say) $\in \Delta(z)$, so that $F^{-}\left(\bigcap_{i=1}^{\infty} W_{i}\right) \in \mathcal{F}$. For any $V \in \Delta(A)$, since $z \in \delta-a d F^{-}(\Delta(A))$, we have $F(V) \cap W \neq \Phi$, i.e., $\Phi \neq V \cap F^{-}(W)=V \cap F^{-}\left(\bigcap_{i=1}^{\infty} W_{i}\right)=V \cap\left(\bigcap_{i=1}^{\infty} F^{-}\left(W_{i}\right)\right)$. Thus by Lemma 4.12, $A \cap \delta-a d F^{-}(\Delta(z)) \neq \Phi$. For each $x \in A \cap \delta-a d F^{-}(\Delta(z))$, we have $V \cap F^{-}(W) \neq \Phi$, and hence $F(V) \cap W \neq \Phi$, for all $V \in \Delta(x)$ and $W \in \Delta(z)$ i.e., $F(V) \cap W \neq \Phi$. Thus $z \in \delta \operatorname{cl} F(V)$, for each $V \in \Delta(x)$. Then $z \in \mathcal{D}(F, x) \subset \mathcal{D}(F, A)$. Consequently, $\delta-\operatorname{ad} F(\Delta(A)) \subset \mathcal{D}(F, A)$. This along with (1) proves the result.

(b) $\Rightarrow$ (c): Clear.

$\left(\right.$ c) $\Rightarrow$ (a): Let $\mathcal{F}$ be a filterbase on $X$ with the countable intersection property. Let $\mathcal{F}^{*}$ denote the filterbase, each of whose members is a countable intersection of members of $\mathcal{F}$. Let $y_{0} \notin X$, and set $Y=X \cup\left\{y_{0}\right\}$ and $\tau_{Y}=\left\{U \subset Y: y_{0} \notin U\right.$ or $F^{*} \subset U$ for some $\left.F^{*} \in \mathcal{F}^{*}\right\}$. Clearly $\tau_{Y}$ is a topology on $Y$. We show that $Y$ is a $D$-space. For this it is sufficient to show that every countable intersection of regular open sets of $Y$ is again regular open, since every $\delta$-open set is a union of some regular open sets. Let $\left\{U_{i}: i=1,2, \ldots\right\}$ be a countable collection of regular open sets of $Y$, and let $U=\bigcap_{i=1}^{\infty} U_{i}$. If $y_{0} \in U$, then $U$ is clearly regular open, as intcl $U=i n t U=U$. So, suppose that $y_{0} \notin V$. Then there is some $U_{n}$ such that $y_{0} \notin U_{n}$. Now, two cases are possible viz. $y_{0} \in \operatorname{cl} U_{n}$ and $y_{0} \notin \operatorname{cl} U_{n}$. If $y_{0} \in \operatorname{cl} U_{n}$, then since $y_{0} \notin U_{n}=$ intcl $U_{n}$, no member of $\mathcal{F}^{*}$, containing $y_{0}$, can be contained in $\operatorname{cl} U_{n}$. Now, $\operatorname{cl} U \subset U \cup\left\{y_{0}\right\}$. Since $\operatorname{cl} U \subset \operatorname{cl} U_{n}$, no member of $\mathcal{F}^{*}$, containing $y_{0}$, can be contained in $\operatorname{cl} U$ and hence $y_{0} \notin \operatorname{intcl} U$, i.e., intcl $U=U$ so that $U$ becomes regular open. In case $y_{0} \notin \operatorname{cl} U_{n}$, we have $y_{0} \notin \mathrm{cl} U$ and hence $c l U=U$. Thus $\operatorname{intcl} U=U$, so that $U$ is regularly open. Thus $\left(Y, \tau_{Y}\right)$ is a $D$-space. 
Now proceeding exactly in the same way as in the proof of '(c) $\Rightarrow$ (a)' of Theorem 4.5, we see that $\delta-a d \mathcal{F}^{*} \neq \Phi$. Clearly $\delta-a d \mathcal{F}^{*} \subset-\delta-a d \mathcal{F}$, and hence $\delta-a d \mathcal{F} \neq \Phi$. The rest follows from Theorem 4.11.

Like Corollary 4.6, we have from the above proof that

Corollary 4.15. Let $X$ and $Y$ be spaces with $Y$ a D-space. If $A(\subset X)$ is nearly Lindelöf relative to $X$, then $\mathcal{D}(F, A)=\delta c l \mathcal{D}(F, A)=\delta-\operatorname{ad}(F(\Delta(A)))$.

Again, from the above Corollary and Theorem 3.5 we obtain:

Corollary 4.16. Let $X$ and $Y$ be spaces with $Y$ a D-space. If $F \in M(X, Y)$ has a $\delta$-closed graph, then $F(A)$ is $\delta$-closed in $Y$ for each nearly Lindelöf set $A$ relative to $X$.

\section{References}

[1] C. K. Basu, "On nearly compact spaces and $\delta^{*}$-continuous function," Boll. Un. Mat. Ital. (to appear).

[2] N. Bourbaki, Elements of Mathematics, General Topology, Part I, Herrmann, Paris, AddisonWesley, Reading, Massachusetts, 1966.

[3] D. A. Carnahan, "Locally nearly compact space," Boll. Un. Mat. Ital. (4) 6, 146-153, 1972.

[4] T. R. Hamlett, "Applications of cluster sets in minimal topological spaces," Proc. Amer. Math. Soc., 53, 477-480, 1975.

[5] T. R. Hamlett, "Cluster sets in general topology," J. London Math. Soc., 12, 192-198, 1976.

[6] L. L. Herrington, "Properties of nearly compact spaces," Proc. Amer. Math. Soc., 45, 431-436, 1974.

[7] J. E. Joseph, "Multifunctions and cluster sets," Proc. Amer. Math. Soc., 74(2), 329-337, 1979.

[8] Asha, Mathur, "Some weaker forms of compactness," Proceedings of the Kanpur Topological Conference, 181-191, 1968.

[9] M. Mršević, I. L. Reilly and M. K. Vamanamurthy, "On nearly Lindelöf spaces," Glasnik Mat., 21(41), 407-414, 1986.

[10] T. Noiri, "A. generalization of perfect function," J. London Math. Soc., 17(2), 540-544, 1978.

[11] T. Noiri, "On $\delta$-continuous functions," J. Korean Math. Soc., 16(2), 161-166, 1980.

[12] M. K. Singal and S. P. Arya, "On almost regular spaces," Glasnik Mat., 4(24), 89-99, 1969.

[13] M. K. Singal and A. Mathur, "On nearly compact spaces," Boll. Un. Mat. Ital., 6(4), 63-73, 1969.

[14] N. V. Veličko, "H-closed topological spaces," Amer. Math. Soc. Transl., 78(2), 103-118, 1968.

[15] J. D. Weston, "Some theorems on cluster sets," J. London Math. Soc., 33, 435-441, 1958.

Department of Pure Mathematics, University of Calcutta, 35-Ballygunge Circular Road, Calcutta-700 019, India. 